\title{
On the monotonicity of the eigenvector method
}

\author{
László Csatóa $^{\mathrm{a}} \quad$ Dóra Gréta Petróczy ${ }^{\mathrm{b}}$
}

21st October 2020

"But nobody should deny the following principle"1

(Edmund Landau: Über Preisverteilung bei Spielturnieren)

\begin{abstract}
Pairwise comparisons are used in a wide variety of decision situations where the importance of alternatives should be measured on a numerical scale. One popular method to derive the priorities is based on the right eigenvector of a multiplicative pairwise comparison matrix. We consider two monotonicity axioms in this setting. First, increasing an arbitrary entry of a pairwise comparison matrix is not allowed to result in a counter-intuitive rank reversal, that is, the favoured alternative in the corresponding row cannot be ranked lower than any other alternative if this was not the case before the change (rank monotonicity). Second, the same modification should not decrease the normalised weight of the favoured alternative (weight monotonicity). Both properties are satisfied by the geometric mean method but violated by the eigenvector method. The axioms do not uniquely determine the geometric mean. The relationship between the two monotonicity properties and the Saaty inconsistency index are investigated for the eigenvector method via simulations. Even though their violation turns out not to be a usual problem even for heavily inconsistent matrices, all decision-makers should be informed about the possible occurrence of such unexpected consequences of increasing a matrix entry.
\end{abstract}

Keywords: Decision analysis; axiomatic approach; eigenvector method; monotonicity; pairwise comparisons

MSC class: 90B50, 91B08

JEL classification number: C44, D71

${ }^{\mathrm{a}}$ Corresponding author. E-mail: laszlo.csato@sztaki.hu

Institute for Computer Science and Control (SZTAKI), Laboratory on Engineering and Management

Intelligence, Research Group of Operations Research and Decision Systems

Corvinus University of Budapest (BCE), Department of Operations Research and Actuarial Sciences

b E-mail: doragreta.petroczy@uni-corvinus.hu

Corvinus University of Budapest (BCE), Department of Finance

${ }^{1}$ In the original: "Niemand aber dürfte folgendes Prinzip bestreiten" (Landau, 1914, p. 201). 


\section{Introduction}

Several decision-making methods involve the comparison of the criteria and the alternatives in pairs, making judgements, and compiling the results into multiplicative positive reciprocal pairwise comparison matrices. For instance, this is a crucial component of the Analytic Hierarchy Process (AHP), introduced by Thomas L. Saaty (Saaty, 1977, 1980). He has suggested deriving the priorities from such a matrix by its principal right eigenvector, which is called the eigenvector method. Since AHP has numerous applications (Ho, 2008; Saaty and Vargas, 2012; Vaidya and Kumar, 2006), a better understanding of this procedure seems to be a prominent research question.

The starting point of our paper is a remark of Saaty (Saaty, 2003, p. 86): "Now we ask the question, what is priority or more generally what meaning should we attach to a priority vector of a set of alternatives? We can think of two meanings. The first is a numerical ranking of the alternatives that indicates an order of preference among them. The other is that the ordering should also reflect intensity or cardinal preference as indicated by the ratios of the numerical values and is thus unique to within a positive multiplicative constant (a similarity transformation)." A potential implication is that both the rank and the (normalised) weight of any alternative should be a monotonic function of its comparisons. In other words, increasing an arbitrary entry in the $i$ th row of a pairwise comparison matrix should not result in a rank reversal such that alternative $i$ was ranked at least as high as alternative $k$ before the change but it is ranked lower after the change. Similarly, such a change is not allowed to decrease the overall importance of alternative $i$. If the straightforward conditions of rank monotonicity or weight monotonicity do not hold, then the ordering or the weights of the alternatives may behave against the intentions of the decision-maker who wants to express a stronger preference for an alternative by increasing its pairwise comparisons.

The eigenvector method will be proved to suffer from both problems for certain pairwise comparison matrices, while the geometric mean method always satisfies these requirements. It is also shown that the axioms do not uniquely determine the geometric mean, hence they can serve as reasonable criteria to classify the weighting methods suggested for pairwise comparison matrices.

It will also be investigated how often the eigenvector method violates these properties for a randomly generated matrix as a function of its consistency ratio, the consistency measure suggested by Saaty (1977). In particular, the axioms are tested by substituting a randomly chosen matrix entry with the next element on the well-known Saaty scale. Their violation turns out not to be a usual problem even for heavily inconsistent matrices. Nonetheless, all decision-makers should be informed about the possible occurrence of counter-intuitive changes after a matrix entry is increased because probably nobody expects that a more favourable opinion on an alternative can be detrimental to its rank or weight.

The paper has the following structure. Section 2 outlines the topic of pairwise comparison matrices and introduces the two axioms of monotonicity. The eigenvector method and some other procedures are analysed in the view of these properties in Section 3. Finally, Section 4 concludes. 


\section{The problem}

In this section, the main notions around pairwise comparison matrices are briefly recalled, and two natural properties are presented. We also offer a short overview of related papers.

\subsection{Preliminaries: multiplicative pairwise comparison matrices}

Let $N=\{1,2, \ldots, n\}$ be a set of alternatives to be evaluated. Assume that their pairwise comparisons are known: $a_{i j}$ is a numerical answer to the question "How many times alternative $i$ is better than alternative $j$ ?", that is, $a_{i j}$ quantifies the relative importance of alternative $i$ with respect to alternative $j$.

Let $\mathbb{R}_{+}^{n}$ and $\mathbb{R}_{+}^{n \times n}$ denote the set of positive (with all elements greater than zero) vectors of size $n$ and matrices of size $n \times n$, respectively.

The comparisons are collected into a matrix whose entries below the diagonal are reciprocal to the corresponding entries above the diagonal.

Definition 2.1. Multiplicative pairwise comparison matrix: Matrix $\mathbf{A}=\left[a_{i j}\right] \in \mathbb{R}_{+}^{n \times n}$ is a multiplicative pairwise comparison matrix if $a_{j i}=1 / a_{i j}$ for all $1 \leq i, j \leq n$.

In the following, the word "multiplicative" will be omitted for the sake of simplicity.

The set of all pairwise comparison matrices with $n$ alternatives is denoted by $\mathcal{A}^{n \times n}$.

Pairwise comparisons are carried out in order to obtain a priority vector $\mathbf{w}$ such that the proportion of the weights $w_{i}$ and $w_{j}$ of the alternatives $i$ and $j$, respectively, approximates the value of their pairwise comparison, that is, $w_{i} / w_{j} \approx a_{i j}$. Thus the weights can be normalised arbitrarily.

Definition 2.2. Weight vector: Vector $\mathbf{w}=\left[w_{i}\right] \in \mathbb{R}_{+}^{n}$ is a weight vector if $\sum_{i=1}^{n} w_{i}=1$.

The set of weight vectors of size $n$ is denoted by $\mathcal{R}^{n}$.

Definition 2.3. Weighting method: Function $f: \mathcal{A}^{n \times n} \rightarrow \mathcal{R}^{n}$ is a weighting method.

The weight of alternative $i$ from the pairwise comparison matrix $\mathbf{A}$ according to the weighting method $f$ is denoted by $f_{i}(\mathbf{A})$.

Weighting methods are often used to rank the alternatives. Ranking $\succeq$ is a weak order on the set of alternatives $N$. Any ranking $\succeq$ has two parts, the asymmetric relation $\succ$, and the symmetric relation $\sim$, defined as follows: $i \succ j$ if and only if $i \succeq j$ but $i \preceq j$ does not hold, and $i \sim j$ if and only if $i \succeq j$ and $i \preceq j$, respectively.

There exist many methods to estimate a suitable weight vector from a pairwise comparison matrix. Probably the most popular procedures are the (row) geometric mean (logarithmic least squares) method (Williams and Crawford, 1980; Crawford and Williams, 1985; De Graan, 1980; de Jong, 1984; Rabinowitz, 1976), and the eigenvector method (Saaty, 1977). Although the latter suffers from a number of theoretical shortcomings discussed later, and there are sound axiomatic arguments in favour of the geometric mean (Fichtner, 1984; Lundy et al., 2017; Csató, 2018; Bozóki and Tsyganok, 2019; Csató, 2019a), the AHP methodology mainly uses the eigenvector method since the pioneering work of Saaty. Therefore, this procedure will be in our focus.

Definition 2.4. Eigenvector method (Saaty, 1977): The eigenvector method associates the weight vector $\mathbf{w}^{E M}(\mathbf{A}) \in \mathcal{R}^{n}$ for a given pairwise comparison matrix $\mathbf{A} \in \mathcal{A}^{n \times n}$ such that

$$
\mathbf{A w}^{E M}(\mathbf{A})=\lambda_{\max }(\mathbf{A}) \mathbf{w}^{E M}(\mathbf{A})
$$


where $\lambda_{\max }(\mathbf{A})$ denotes the maximal eigenvalue, also known as the principal or Perron eigenvalue, of the (positive) matrix $\mathbf{A}$.

Definition 2.5. Geometric mean method (Williams and Crawford, 1980; Crawford and Williams, 1985; De Graan, 1980; de Jong, 1984; Rabinowitz, 1976): The geometric mean method associates the weight vector $\mathbf{w}^{G M}(\mathbf{A}) \in \mathcal{R}^{n}$ for a given pairwise comparison matrix $\mathrm{A} \in \mathcal{A}^{n \times n}$, where

$$
w_{i}^{G M}(\mathbf{A})=\frac{\prod_{j=1}^{n} a_{i j}^{1 / n}}{\sum_{k=1}^{n} \prod_{j=1}^{n} a_{k j}^{1 / n}} .
$$

Another priority deriving method is considered to illustrate monotonicity.

Definition 2.6. Column sum method (Zahedi, 1986; Choo and Wedley, 2004): The column sum method associates the weight vector $\mathbf{w}^{C M}(\mathbf{A}) \in \mathcal{R}^{n}$ for a given pairwise comparison matrix $\mathbf{A} \in \mathcal{A}^{n \times n}$, where

$$
w_{i}^{C M}(\mathbf{A})=\frac{\sum_{j=1}^{n} a_{i j}}{\sum_{k=1}^{n} \sum_{j=1}^{n} a_{k j}} .
$$

All weighting methods induce a ranking, for instance, $i \succeq_{\mathbf{A}}^{E M} j$ if and only if $w_{i}^{E M}(\mathbf{A}) \geq$ $w_{j}^{E M}(\mathbf{A})$.

There is a special case when all reasonable weighting methods, including the above three, give the same result.

Definition 2.7. Consistency: Let $\mathbf{A}=\left[a_{i j}\right] \in \mathbb{R}_{+}^{n \times n}$ be a pairwise comparison matrix. It is called consistent if the condition $a_{i k}=a_{i j} a_{j k}$ holds for all $1 \leq i, j, k \leq n$.

However, consistency is seldom observed in practice, pairwise comparison matrices are usually inconsistent. A variety of indices has been proposed to measure the level of inconsistency, see Brunelli (2018) for a survey of them. We will consider the oldest and by far the most popular Saaty inconsistency index (Saaty, 1977), which is closely related to the eigenvector method.

Definition 2.8. Consistency index $(C I)$ : Let $\mathbf{A}=\left[a_{i j}\right] \in \mathbb{R}_{+}^{n \times n}$ be a pairwise comparison matrix. Its consistency index is

$$
C I(\mathbf{A})=\frac{\lambda_{\max }(\mathbf{A})-n}{n-1}
$$

where $\lambda_{\max }(\mathbf{A})$ is the principal eigenvalue of matrix $\mathbf{A}$ as before.

Saaty (1977) introduced the so-called random index $R I_{n}$, that is, the average $C I$ of a large number of $n \times n$ pairwise comparison matrices with entries randomly generated from the scale $\{1 / 9,1 / 8, \ldots, 8,9\}$. The proportion of $C I$ and $R I_{n}$ is called the consistency ratio $C R$, or the Saaty inconsistency index.

Saaty (1977) considered a pairwise comparison matrix to be acceptable if the value of $C R$ does not exceed the threshold 0.1 .

\subsection{Monotonicity on single comparisons}

The entry $a_{i j}$ measures the dominance of alternative $i$ over alternative $j$, thus it is not expected that increasing $a_{i j}$ leads to an ordering where alternative $i$ is ranked lower than any alternative $k$ if it was ranked at least as high before the change. Such counter-intuitive rank reversal would be probably against the intention of the decision-maker.

The following axiom formalises this requirement. 
Axiom 1. Rank monotonicity: Let $\mathbf{A} \in \mathcal{A}^{n \times n}$ be any pairwise comparison matrix and $1 \leq i, j \leq n$ be any two different alternatives. Let $\mathbf{A}^{\prime} \in \mathcal{A}^{n \times n}$ be identical to $\mathbf{A}$ but $a_{i j}^{\prime}>a_{i j}$ (and $a_{j i}^{\prime}<a_{j i}$ due to the reciprocity property). The weighting method $f: \mathcal{A}^{n \times n} \rightarrow \mathcal{R}^{n}$ is called rank monotonic if $i \succeq_{\mathbf{A}}^{f} k \Rightarrow i \succeq_{\mathbf{A}^{\prime}}^{f} k$ for all $1 \leq k \leq n$.

Rank monotonicity is widely considered in social choice theory, hence it would be difficult to give credit to any author for first suggesting this property. Rubinstein (1980) uses it to characterise the ranking method commonly known as the points system on the set of tournaments, where either player $i$ beats player $j$, or player $j$ beats player $i$. Henriet (1985) extends this result by dropping the asymmetric property of the beating relation, which is further generalised by Bouyssou (1992) to the case of valued relations. The axiom is called positive responsiveness in van den Brink and Gilles (2009) and positive responsiveness to the beating relation in González-Díaz et al. (2014). Analogously, Boldi et al. (2017) examine the rank monotonicity of centrality measures with respect to adding a new arc to a network.

A stronger version has been used in the axiomatic characterization of the ranking induced by the geometric mean (Csató, 2018): $i \succeq j$ implies $i \succ j$ whenever $a_{i j}$ increases. According to Bouyssou and Perny (1992, p. 188), rank monotonicity seems an unobjectionable property in the context of partial ranking methods but its stronger version is much more demanding.

In some applications, the weights of the alternatives are more important than their ranking because, for example, certain resources will be allocated on the basis of the weights. Then it makes sense to exclude the possibility that the weight of any alternative decreases after one of its pairwise comparisons increases.

Axiom 2. Weight monotonicity: Let $\mathbf{A} \in \mathcal{A}^{n \times n}$ be any pairwise comparison matrix and $1 \leq i, j \leq n$ be any two different alternatives. Let $\mathbf{A}^{\prime} \in \mathcal{A}^{n \times n}$ be identical to $\mathbf{A}$ but $a_{i j}^{\prime}>a_{i j}$ (and $a_{j i}^{\prime}<a_{j i}$ due to the reciprocity property). The weighting method $f: \mathcal{A}^{n \times n} \rightarrow \mathcal{R}^{n}$ is called weight monotonic if

$$
a_{i j}^{\prime}>a_{i j} \Rightarrow \frac{f_{i}\left(\mathbf{A}^{\prime}\right)}{\sum_{k=1}^{n} f_{k}\left(\mathbf{A}^{\prime}\right)} \geq \frac{f_{i}(\mathbf{A})}{\sum_{k=1}^{n} f_{k}(\mathbf{A})} \Longleftrightarrow f_{i}\left(\mathbf{A}^{\prime}\right) \geq f_{i}(\mathbf{A}),
$$

Landau (1914, p. 201) shows that weight monotonicity is violated by the principal right eigenvector for nonnegative matrices of order $n=3$. The interesting and often neglected early observation is commented in David and Edwards (2001, p. 164) with the following words: "This leads Landau to dismiss the Kendall-Wei approach 40 years prior to its birth!" However, the set of pairwise comparison matrices is a strict subset of nonnegative matrices, and the example of Landau is not a pairwise comparison matrix.

Naturally, this type of monotonicity is a standard requirement in many fields of research such as cooperative game theory (Young, 1985), scientometrics (Kóczy and Strobel, 2009; Bouyssou and Marchant, 2014; Perry and Reny, 2016), the theoretical analysis of sports rules (Kendall and Lenten, 2017; Vaziri et al., 2018; Csató, 2019b, 2020a,b), or voting theory (Balinski and Young, 2001; Tasnádi, 2008).

In the context of inconsistency indices, Brunelli and Fedrizzi (2015) have suggested an axiom with the same flavour called monotonicity on single comparisons, which formalizes a property proved by Aupetit and Genest (1993) for the Saaty inconsistency index. The authors also provide a short overview of its origin in multi-criteria decision-making. 


\subsection{Related works}

The current paper is not the first work focusing on a mathematical shortcoming of the eigenvector method. Johnson et al. (1979) argue that the use of the left eigenvector is equally justified as long as the order is reversed, furthermore, the rankings from the two eigenvectors may disagree even when the matrix is nearly consistent. According to Genest et al. (1993), the ordering obtained from the principal right eigenvector depends on the choice of the parameter for numerically coded ordinal preferences. Bana e Costa and Vansnick (2008) find that the right eigenvector can violate a condition of order preservation, which is fundamental in decision aiding according to the authors. Kułakowski (2015) examines the relationship between this property and the inconsistency index proposed by Saaty. Pérez and Mokotoff (2016) present an example where the alternative with the highest priority for all decision-makers is not the best on the basis of their aggregated preferences. Csató (2017) traces back the origin of this problem to the right-left asymmetry (Johnson et al., 1979), and provides a minimal counterexample with four alternatives.

The eigenvector solution is not necessarily Pareto efficient, in other words, there may exist a weight vector which is at least as good in approximating all elements of the pairwise comparison matrix, and strictly better in at least one position (Blanquero et al., 2006). Ábele-Nagy and Bozóki (2016) prove that it is not possible if the pairwise comparison matrix differs from a consistent one only in one entry (and its reciprocal), while Ábele-Nagy et al. (2018) extend this result to double perturbed matrices, which can be made consistent by altering two elements and their reciprocals. On the other hand, the eigenvector method may lead to an inefficient weight vector for matrices with an arbitrarily small inconsistency (Bozóki, 2014). Bozóki and Fülöp (2018) propose linear programs to test whether a given weight vector is efficient or not, and Duleba and Moslem (2019) give the first examination of this property on real data.

\section{Results}

The row geometric mean (logarithmic least squares) method trivially satisfies monotonicity: a greater value of $a_{i j}$ increases the weight of alternative $i$, decreases the weight of alternative $j$, while preserves the weights of all other alternatives before normalisation. The case of the eigenvector method turns out to be more complicated.

\subsection{The geometric mean method is monotonic}

According to the following result, some weighting methods meet Axioms 1 and 2.

Proposition 3.1. The geometric mean and column sum methods satisfy both rank monotonicity and weight monotonicity.

Proof. Consider the pairwise comparison matrices $\mathbf{A}$ and $\mathbf{A}^{\prime}$, which are identical except for $a_{i j}^{\prime}>a_{i j}$ and $a_{j i}^{\prime}<a_{j i}$.

Geometric mean method: $i \succeq_{\mathbf{A}}^{G M} k$ means that $\prod_{\ell=1}^{n} a_{i \ell} \geq \prod_{\ell=1}^{n} a_{k \ell}$. Since $\prod_{\ell=1}^{n} a_{i \ell}^{\prime}>$ $\prod_{\ell=1}^{n} a_{i \ell}$ and $\prod_{\ell=1}^{n} a_{k \ell}^{\prime} \leq \prod_{\ell=1}^{n} a_{k \ell}$ if $k \neq i, \prod_{\ell=1}^{n} a_{i \ell}^{\prime}>\prod_{\ell=1}^{n} a_{k \ell}^{\prime}$, which implies $i \succ_{\mathbf{A}^{\prime}}^{G M} k$, that is, rank monotonicity. As only the weight of alternative $i$ increases, weight monotonicity holds, too.

Column sum method: $i \succeq_{\mathbf{A}}^{C M} k$ means that $\sum_{\ell=1}^{n} a_{i \ell} \geq \sum_{\ell=1}^{n} a_{k \ell}$. Since $\sum_{\ell=1}^{n} a_{i \ell}^{\prime}>$ $\sum_{\ell=1}^{n} a_{i \ell}$ and $\sum_{\ell=1}^{n} a_{k \ell}^{\prime} \leq \sum_{\ell=1}^{n} a_{k \ell}$ if $k \neq i, \sum_{\ell=1}^{n} a_{i \ell}^{\prime}>\sum_{\ell=1}^{n} a_{k \ell}^{\prime}$, which implies $i \succ_{\mathbf{A}^{\prime}}^{C M} k$, that 
Figure 1: Weights derived by the eigenvector method in Example 3.1

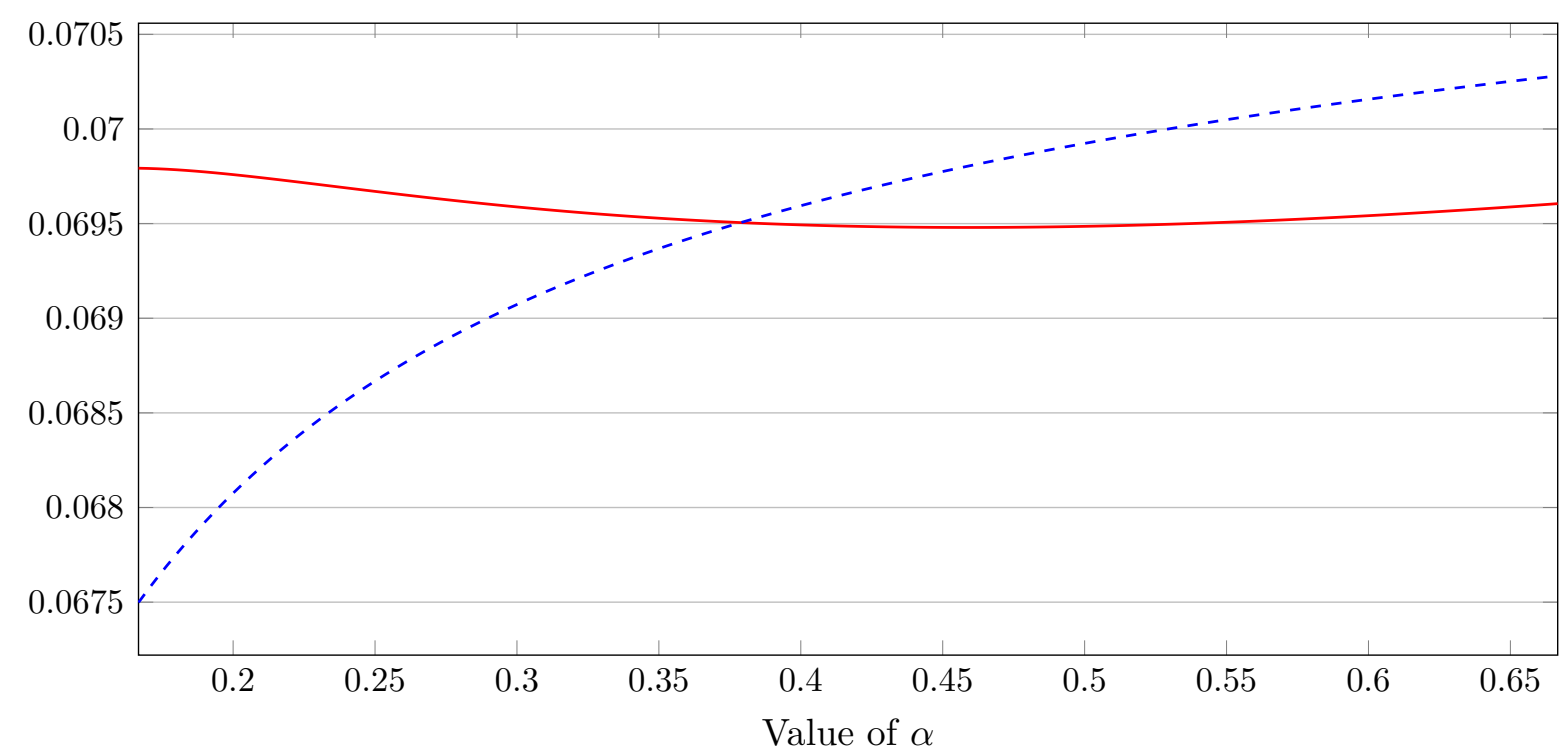

- The weight $w_{1}^{E M}\left(\mathbf{A}^{\alpha}\right)$ of the first alternative

- - - The weight $w_{5}^{E M}\left(\mathbf{A}^{\alpha}\right)$ of the fifth alternative

is, rank monotonicity. As only the weight of alternative $i$ increases, weight monotonicity holds, too.

Csató (2018, Proposition 2) has already shown the rank monotonicity of geometric mean.

\subsection{The eigenvector method violates monotonicity}

However, not all weighting methods are compatible with Axioms 1 and 2.

Proposition 3.2. The eigenvector method does not satisfy rank monotonicity.

Proof. It is sufficient to provide a counterexample.

Example 3.1. Consider the following parametric pairwise comparison matrix:

$$
\mathbf{A}^{\alpha}=\left[\begin{array}{cccccc}
1 & \alpha & 8 & 1 / 9 & 1 / 4 & 1 / 9 \\
1 / \alpha & 1 & 1 / 5 & 1 / 9 & 1 & 1 / 4 \\
1 / 8 & 5 & 1 & 1 / 8 & 1 / 2 & 1 / 7 \\
9 & 9 & 8 & 1 & 7 & 8 \\
4 & 1 & 2 & 1 / 7 & 1 & 1 / 9 \\
9 & 4 & 7 & 1 / 8 & 9 & 1
\end{array}\right]
$$

The weights of the first and the fifth alternatives are plotted in Figure 1 as a function of parameter $\alpha$.

Note that the fifth alternative becomes more important than the first when $a_{12}$ is increased from 0.3 to 0.5 , showing the violation of rank monotonicity by the eigenvector method. 
What does cause the violation of rank monotonicity in Example 3.1? The growth of $\alpha$ increases $w_{1}$, which is also favourable for $w_{5}$ since $w_{1} / w_{5} \approx a_{15}=1 / 4$. On the other hand, there is a „normalisation effect”, which decreases $w_{5}$ as the sum of all weights is fixed.

Proposition 3.3. The eigenvector method does not satisfy weight monotonicity.

Proof. Consider Example 3.1. According to Figure 1, the normalised weight of the first alternative has a minimum around $\alpha \approx 0.5$, thus the eigenvector method does not satisfy weight monotonicity.

It remains difficult to explain the lack of weight monotonicity due to the multiple connections between the weights of alternatives as revealed by the formula $w_{i}^{E M}=$ $\lambda_{\max } \sum_{\ell=1}^{n} a_{i \ell} w_{\ell}^{E M}$. Unfortunately, this is a common phenomenon for the unfavourable properties of the eigenvector method (Johnson et al., 1979; Blanquero et al., 2006; Bana e Costa and Vansnick, 2008).

As it has already been mentioned, the principal right eigenvector violates Axiom 2 on the domain of nonnegative matrices of order $n=3$ (Landau, 1914, p. 201). A section titled "Non-monotonicity of some weight extraction methods" on the Wikipedia page of the Analytic Hierarchy Process says that the eigenvector method is non-monotonic for reciprocal $n \times n$ matrices where $n>3$. This contribution has been added on 10 December 2013 by the Dutch international draughts (dammen) player Kees Pippel. We have managed to consult him, and he confirmed that it refers to the example of Landau, which does not imply Proposition 3.3 due to the different domain.

\subsection{A framework for analysing the two axioms of monotonicity}

In order to investigate the probability of a non-monotonic eigenvector, we apply a computational technique and consider a large number of pairwise comparison matrices that are checked for both properties.

The entries of the random pairwise comparison matrices are generated according to the standard proposed by Saaty, which has also been used, for example, in Bozóki and Rapcsák (2008). Thus all entries $a_{i j}$ above the diagonal $(i<j)$ are randomly chosen from the discrete set

$$
\left\{\frac{1}{9} ; \frac{1}{8} ; \frac{1}{7} ; \frac{1}{6} ; \frac{1}{5} ; \frac{1}{4} ; \frac{1}{3} ; \frac{1}{2} ; 1 ; 2 ; 3 ; 4 ; 5 ; 6 ; 7 ; 8 ; 9\right\}
$$

with equal probability $1 / 17$, and by setting $a_{j i}=1 / a_{i j}$, as well as $a_{i i}=1$.

Remark 1 . The monotonicity of the principal right eigenvector is tested by substituting a matrix entry with the next element from the scale (5), that is, if $2 \leq a \leq 9, a \in \mathbb{N}$, then $a_{i j}=1 / a$ is substituted with $1 /(a-1)$, and if $1 \leq b \leq 9, b \in \mathbb{N}$, then $a_{i j}=b$ is substituted with $b+1$. This seems to be a realistic scenario since the decision-maker probably does not expect a counter-intuitive change in the ranking or weights when thinking over to give a more favourable opinion on an alternative.

Consequently, one iteration of the computational process consists of the following steps:

1. A random pairwise comparison matrix $\mathbf{A}$ of order $n$ is generated on the Saaty scale (5).

2. Its consistency ratio $C R(\mathbf{A})$ and eigenvector $\mathbf{w}^{E M}(\mathbf{A})$ is calculated, the number of matrices in the $m$ th interval of consistency ratios, for which $0.01(m-1) \leq$ $C R(\mathbf{A})<0.01 m$, is increased by one. 
3. All entries above the diagonal are considered separately, hence $n(n-1) / 2$ perturbed pairwise comparison matrices $\mathbf{A}^{i j}$ are introduced such that $\mathbf{A}^{i j}=\mathbf{A}$ except for its element in the $i$ th row and $j$ th column, $i<j$. This value is increased to $a_{i j}^{i j}$ following Remark 1, while reciprocity is preserved, thus $a_{j i}^{i j}=1 / a_{i j}^{i j}$.

4. The eigenvectors $\mathbf{w}\left(\mathbf{A}^{i j}\right)$ are computed.

5. Monotonicity check:

- Rank monotonicity: $w_{i}^{E M}(\mathbf{A}) / w_{k}^{E M}(\mathbf{A})$ and $w_{i}^{E M}\left(\mathbf{A}^{i j}\right) / w_{k}^{E M}\left(\mathbf{A}^{i j}\right)$ are compared for all $i<j$ and $1 \leq k \leq n$.

The $m$ th interval of consistency ratios with the flag of rank monotonicity violation, in which $C R(\mathbf{A})$ falls, is increased by one if $w_{i}^{E M}(\mathbf{A})>w_{k}^{E M}(\mathbf{A})$ but $w_{i}^{E M}\left(\mathbf{A}^{i j}\right)<w_{k}^{E M}\left(\mathbf{A}^{i j}\right)$, that is, Axiom 1 is not satisfied after increasing $a_{i j}$ on the scale (5).

- Weight monotonicity: $w_{i}^{E M}(\mathbf{A})$ and $w_{i}^{E M}\left(\mathbf{A}^{i j}\right)$ are compared for all $i<j$.

The $m$ th interval of consistency ratios with the flag of weight monotonicity violation, in which $C R(\mathbf{A})$ falls, is increased by one if $w_{i}^{E M}(\mathbf{A})>$ $w_{i}^{E M}\left(\mathbf{A}^{i j}\right)$, that is, Axiom 2 is not satisfied after increasing $a_{i j}$ on the scale (5).

6. The pairwise comparison matrix $\mathbf{A}$, its consistency ratio $C R(\mathbf{A})$ and $i, j, k$ are saved as an example violating rank monotonicity, weight monotonicity, or both of them, if $C R(\mathbf{A})$ is smaller than the consistency ratio of all previously generated pairwise comparison matrices, where the eigenvector does not satisfy rank monotonicity, weight monotonicity, or both of them, respectively.

Steps 1-6 are repeated until the number of randomly generated pairwise comparison matrices reaches a predetermined limit.

Table 1: Values of the random index used in the computations

\begin{tabular}{lccccccc}
\hline Matrix size & 4 & 5 & 6 & 7 & 8 & 9 & 10 \\
\hline Random index $R I_{n}$ & 0.884 & 1.109 & 1.249 & 1.341 & 1.404 & 1.451 & 1.486 \\
\hline
\end{tabular}

The random indices $R I_{n}$ for the calculation of the consistency ratio $C R$ are reported in Table 1 for $4 \leq n \leq 10$. They are imported from Bozóki and Rapcsák (2008, Table 3) and have been validated by our simulations, too. According to Saaty and Ozdemir (2003), it is reasonable to compare until nine items, hence we have not examined matrices with more than ten alternatives.

\subsection{The connection of monotonicity and inconsistency}

Remark 2. The eigenvector method satisfies both axioms of monotonicity if the number of alternatives is three because it is equivalent to the geometric mean method for $n=3$ (Crawford and Williams, 1985), and Proposition 3.1 can be applied. 
For $n=4$, there are only six elements above the diagonal, hence the total number of different matrices using the scale (5) is $17^{6}=24,137,569$. We have checked all of them through the process described in steps 1-6, and have not found any violation of monotonicity by the eigenvector method. On the other hand, there are some cases where this problem emerges if the number of alternatives is at least five. Therefore, 10 million randomly generated matrices have been examined for $5 \leq n \leq 8$, as well as 5 million for $9 \leq n \leq 10$.

Figure 2 plots the proportion of pairwise comparison matrices for which the eigenvector method does not satisfy Axiom 1 or Axiom 2 as the function of the consistency ratio $C R$. Note that $C R$ cannot be arbitrarily large: Aupetit and Genest (1993) derive a sharp upper bound on $\lambda_{\max }$ when the responses are coded on a bounded scale applied here.

The probability of violating rank monotonicity almost linearly increases when the pairwise comparison matrix becomes more inconsistent. There is no violation of Axiom 1 for nearly consistent matrices $(C I<0.2)$, and the problem emerges only with a probability of around $2 \%$ even for heavily inconsistent matrices.

Surprisingly, this is not the case for weight monotonicity, Axiom 2 is violated primarily by matrices having a consistency ratio of about 0.5 . Weight monotonicity is satisfied more frequently than rank monotonicity for matrices of order 5 and 6 , however, this result does not hold for moderately inconsistent matrices when $n \geq 7$.

Table 2: The frequency of violating monotonicity properties by the eigenvector method

(a) All values of $C R$

\begin{tabular}{ccccc}
\hline Matrix size & Sample size & \multicolumn{2}{c}{ Ratio of matrices } & Number of matrices violating \\
& & Rank & Weight & both monotonicity properties \\
\hline 5 & $10^{7}$ & $0.47 \%$ & $0.003 \%$ & 0 \\
6 & $10^{7}$ & $0.66 \%$ & $0.070 \%$ & 2 \\
7 & $10^{7}$ & $0.80 \%$ & $0.077 \%$ & 11 \\
8 & $10^{7}$ & $0.91 \%$ & $0.046 \%$ & 7 \\
9 & $5 \times 10^{6}$ & $1.00 \%$ & $0.020 \%$ & 2 \\
10 & $5 \times 10^{6}$ & $1.05 \%$ & $0.007 \%$ & 0 \\
\hline
\end{tabular}

(b) $C R<0.5$

\begin{tabular}{crccc}
\hline Matrix size & Sample size & \multicolumn{2}{c}{ Number of matrices violating monotonicity } \\
& & Rank & Weight & Both \\
\hline 5 & $16,916,343$ & 1,146 & 0 & 0 \\
6 & $7,353,667$ & 2,665 & 313 & 1 \\
7 & $2,712,997$ & 2,295 & 1,701 & 2 \\
8 & 827,413 & 1,059 & 1,307 & 6 \\
9 & 207,424 & 303 & 435 & 2 \\
10 & 41,826 & 80 & 110 & 0 \\
\hline
\end{tabular}

As Example 3.1 shows, there are also some matrices for which the eigenvector method violates both rank and weight monotonicity simultaneously. Since these cases are rare, they are not plotted in Figure 2 but summarised in Table 2. The latter also reinforces the message of Figure 2: the violation of rank monotonicity becomes more serious as the 
Figure 2: Randomly generated pairwise comparison matrices for which the eigenvector method does not satisfy monotonicity

Matrix size: $n=5$; sample size: $10^{7}$

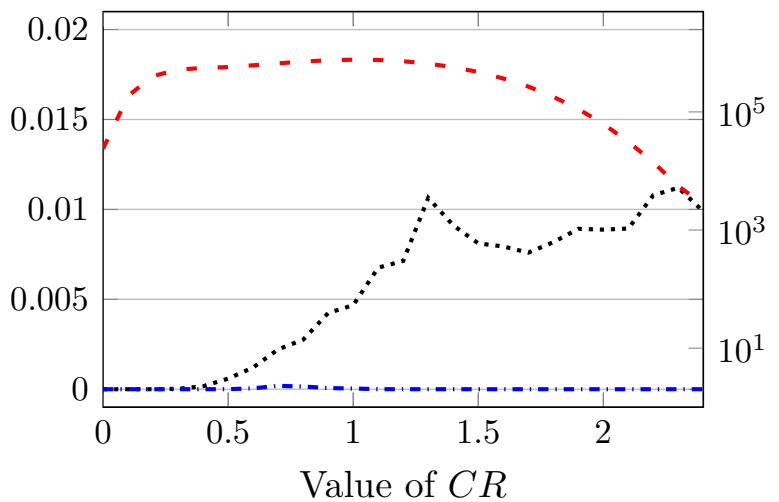

Matrix size: $n=7$; sample size: $10^{7}$

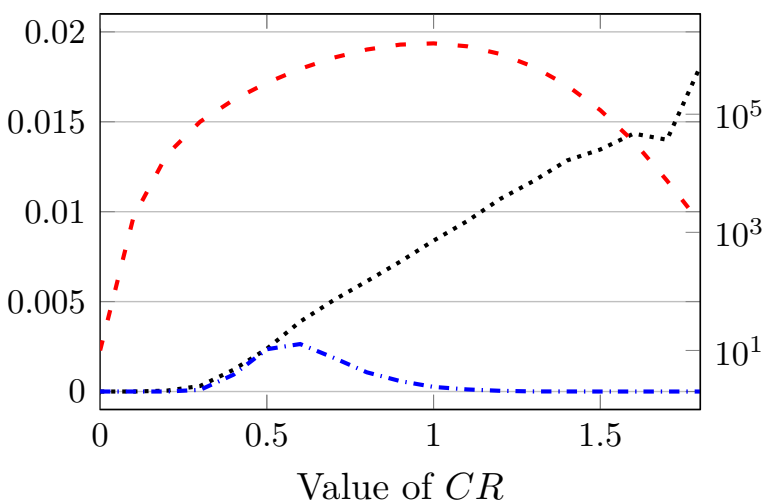

Matrix size: $n=9$; sample size: $5 \times 10^{6}$

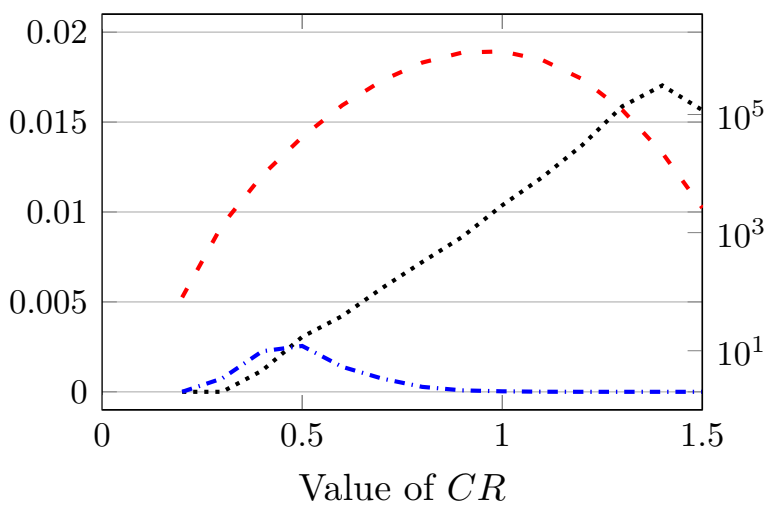

Matrix size: $n=6$; sample size: $10^{7}$

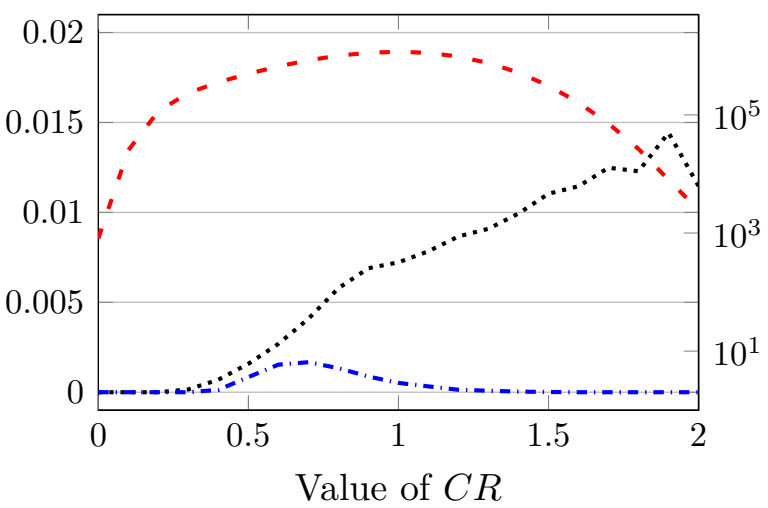

Matrix size: $n=8$; sample size: $10^{7}$

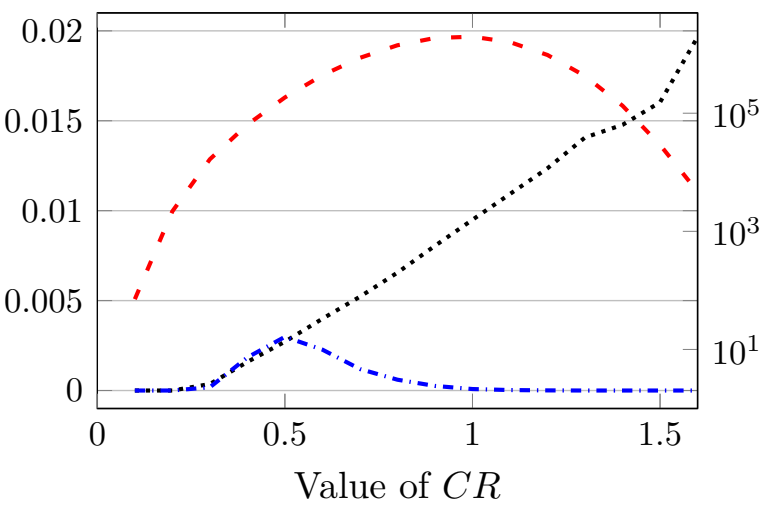

Matrix size: $n=10$; sample size: $5 \times 10^{6}$

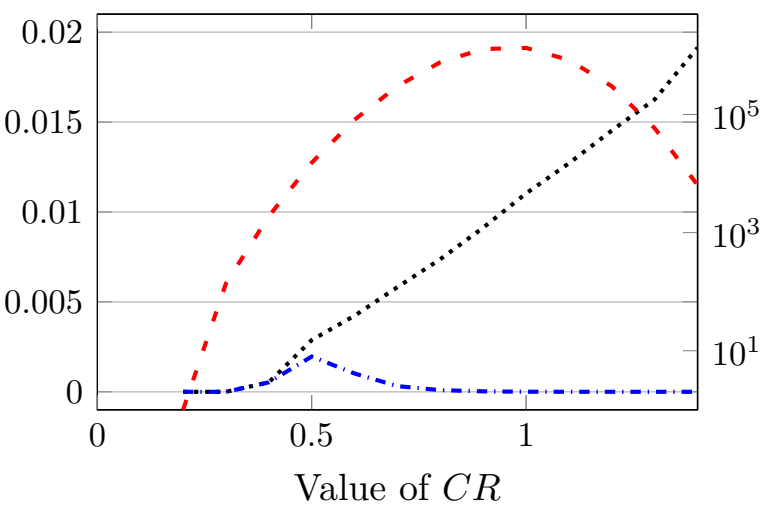

..... Ratio of matrices exhibiting a violation of rank monotonicity (left scale)

- . - Ratio of matrices exhibiting a violation of weight monotonicity (left scale)

- - Number of all randomly generated matrices (right log scale)

number of alternatives increases, while weight monotonicity is the most problematic for $n=7$ if one focuses on all randomly generated matrices.

By limiting the monotonicity check to $C R<0.5$, the number of randomly generated 
matrices can be increased to 100 million. However, only a fraction of them will be moderately inconsistent, see the column sample size in Table 2.b. Then weight monotonicity becomes a more serious issue than rank monotonicity if $n \geq 8$. Furthermore, even the probability of violating weight monotonicity grows along with the size of the matrix for these moderately inconsistent matrices.

The two axioms are clearly independent as revealed by Figure 2. For large values of the consistency ratio $C R$, there is no violation of weight monotonicity (Axiom 2) but there are several violations of rank monotonicity (Axiom 1). On the other hand, it can be seen that weight monotonicity is violated more frequently compared to rank monotonicity for moderate levels of $C R$ if $n=9$. That is reinforced by Table 2.b, where there are more instances of violating rank monotonicity than weight monotonicity if $5 \leq n \leq 7$, however, this relation is reversed if $8 \leq n \leq 10$. The relationship between the two properties is unsurprising in the view of their definitions. Firstly, it might happen that the (normalised) weight of alternative $i$ increases but the (normalised) weight of alternative $j$ grows faster after the entry $a_{i j}$ is increased. Secondly, it might happen that the (normalised) weight of alternative $i$ decreases if $a_{i j}$ is increased, without causing a rank reversal among the alternatives.

\section{Conclusions}

In the current paper, we have argued that two axioms of monotonicity on the numerical comparisons - called rank and weight monotonicity, respectively - are key requirements for any priority vector derived from a pairwise comparison matrix. The eigenvector method is proved to violate both properties. However, contrary to the right-left asymmetry (Johnson et al., 1979) and Pareto inefficiency (Bozóki, 2014), as well as, to the condition of order preservation (Bana e Costa and Vansnick, 2008), the emergence of a problematic situation seems to be avoidable for low levels of inconsistency.

Potential users should be informed about the rare occurrence of counter-intuitive changes in the weights after a matrix entry is increased. Nonetheless, it remains difficult to verify that there is no violation of rank or weight monotonicity in a specific application. Perhaps one can develop an online tool to check these properties, similarly to pcmc.online, which is able to test the Pareto efficiency of the weights derived by the eigenvector method. The interaction with the decision-maker is indispensable: if the violation of monotonicity is judged problematic, the eigenvector method should be exchanged for the row geometric mean. It seems also probable that the violation of rank or weight monotonicity indicates some discrepancies in the data as shown by the lack of the problem at low levels of inconsistency. For instance, the fourth alternative dominates in Example 3.1.

To summarise, our findings have important implications for practitioners. First, they emphasise the need for inconsistency reduction methods (Abel et al., 2018; Aguarón et al., 2021; Bozóki et al., 2015; Ergu et al., 2011). Second, the possibly strange behaviour of the right eigenvector makes the use of this method questionable for inherently inconsistent matrices such as the ones emerging in sports applications (Bozóki et al., 2016; Chao et al., 2018), where rewarding players or teams for poor performance is unfair (Kendall and Lenten, 2017; Vaziri et al., 2018). Third, the violation of monotonicity by the eigenvector method serves as a powerful argument for preferring the use of the geometric mean (logarithmic least squares) method in applications of AHP. 


\section{Acknowledgements}

László Csató, the father of the first author has made a substantial contribution to the paper by helping to code the computations in Python.

We are grateful to Sándor Bozóki, János Fülöp, Tamás Halm, Kees Pippel, and Balázs Vida for useful advice.

The research was supported by the MTA Premium Postdoctoral Research Program grant PPD2019-9/2019, the NKFIH grant K 128573, and the ÚNKP-19-3-III-BCE-97 New National Excellence Program of the Ministry for Innovation and Technology.

\section{References}

Abel, E., Mikhailov, L., and Keane, J. (2018). Inconsistency reduction in decision making via multi-objective optimisation. European Journal of Operational Research, 267(1):212226.

Ábele-Nagy, K. and Bozóki, S. (2016). Efficiency analysis of simple perturbed pairwise comparison matrices. Fundamenta Informaticae, 144(3-4):279-289.

Ábele-Nagy, K., Bozóki, S., and Rebák, Ö. (2018). Efficiency analysis of double perturbed pairwise comparison matrices. Journal of the Operational Research Society, 69(5):707713.

Aguarón, J., Escobar, M. T., and Moreno-Jiménez, J. M. (2021). Reducing inconsistency measured by the Geometric Consistency Index in the Analytic Hierarchy Process. European Journal of Operational Research, 288(2):576-583.

Aupetit, B. and Genest, C. (1993). On some useful properties of the Perron eigenvalue of a positive reciprocal matrix in the context of the analytic hierarchy process. European Journal of Operational Research, 70(2):263-268.

Balinski, M. L. and Young, H. P. (2001). Fair representation: meeting the ideal of one man, one vote. Brookings Institution Press, Washington, D. C., second edition.

Bana e Costa, C. A. and Vansnick, J.-C. (2008). A critical analysis of the eigenvalue method used to derive priorities in AHP. European Journal of Operational Research, 187(3):1422-1428.

Blanquero, R., Carrizosa, E., and Conde, E. (2006). Inferring efficient weights from pairwise comparison matrices. Mathematical Methods of Operations Research, 64(2):271-284.

Boldi, P., Luongo, A., and Vigna, S. (2017). Rank monotonicity in centrality measures. Network Science, 5(4):529-550.

Bouyssou, D. (1992). Ranking methods based on valued preference relations: A characterization of the net flow method. European Journal of Operational Research, 60(1):61-67.

Bouyssou, D. and Marchant, T. (2014). An axiomatic approach to bibliometric rankings and indices. Journal of Informetrics, 8(3):449-477. 
Bouyssou, D. and Perny, P. (1992). Ranking methods for valued preference relations: A characterization of a method based on leaving and entering flows. European Journal of Operational Research, 61(1-2):186-194.

Bozóki, S. (2014). Inefficient weights from pairwise comparison matrices with arbitrarily small inconsistency. Optimization, 63(12):1893-1901.

Bozóki, S., Csató, L., and Temesi, J. (2016). An application of incomplete pairwise comparison matrices for ranking top tennis players. European Journal of Operational Research, 248(1):211-218.

Bozóki, S. and Fülöp, J. (2018). Efficient weight vectors from pairwise comparison matrices. European Journal of Operational Research, 264(2):419-427.

Bozóki, S., Fülöp, J., and Poesz, A. (2015). On reducing inconsistency of pairwise comparison matrices below an acceptance threshold. Central European Journal of Operations Research, 23(4):849-866.

Bozóki, S. and Rapcsák, T. (2008). On Saaty's and Koczkodaj's inconsistencies of pairwise comparison matrices. Journal of Global Optimization, 42(2):157-175.

Bozóki, S. and Tsyganok, V. (2019). The (logarithmic) least squares optimality of the arithmetic (geometric) mean of weight vectors calculated from all spanning trees for incomplete additive (multiplicative) pairwise comparison matrices. International Journal of General Systems, 48(4):362-381.

Brunelli, M. (2018). A survey of inconsistency indices for pairwise comparisons. International Journal of General Systems, 47(8):751-771.

Brunelli, M. and Fedrizzi, M. (2015). Axiomatic properties of inconsistency indices for pairwise comparisons. Journal of the Operational Research Society, 66(1):1-15.

Chao, X., Kou, G., Li, T., and Peng, Y. (2018). Jie Ke versus AlphaGo: A ranking approach using decision making method for large-scale data with incomplete information. European Journal of Operational Research, 265(1):239-247.

Choo, E. U. and Wedley, W. C. (2004). A common framework for deriving preference values from pairwise comparison matrices. Computers \& Operations Research, 31(6):893-908.

Crawford, G. and Williams, C. (1985). A note on the analysis of subjective judgment matrices. Journal of Mathematical Psychology, 29(4):387-405.

Csató, L. (2017). Eigenvector Method and rank reversal in group decision making revisited. Fundamenta Informaticae, 156(2):169-178.

Csató, L. (2018). Characterization of the row geometric mean ranking with a group consensus axiom. Group Decision and Negotiation, 27(6):1011-1027.

Csató, L. (2019a). A characterization of the Logarithmic Least Squares Method. European Journal of Operational Research, 276(1):212-216.

Csató, L. (2019b). UEFA Champions League entry has not satisfied strategyproofness in three seasons. Journal of Sports Economics, 20(7):975-981. 
Csató, L. (2020a). The incentive (in)compatibility of group-based qualification systems. International Journal of General Systems, 49(4):374-399.

Csató, L. (2020b). The UEFA Champions League seeding is not strategy-proof since the 2015/16 season. Annals of Operations Research, 292(1):161-169.

David, H. A. and Edwards, A. W. F. (2001). Annotated Readings in the History of Statistics. Springer Science+Business Media.

De Graan, J. G. (1980). Extensions of the multiple criteria analysis method of T. L. Saaty. Report, National Institute for Water Supply, Voorburg.

de Jong, P. (1984). A statistical approach to Saaty's scaling method for priorities. Journal of Mathematical Psychology, 28(4):467-478.

Duleba, S. and Moslem, S. (2019). Examining Pareto optimality in analytic hierarchy process on real Data: An application in public transport service development. Expert Systems with Applications, 116:21-30.

Ergu, D., Kou, G., Peng, Y., and Shi, Y. (2011). A simple method to improve the consistency ratio of the pair-wise comparison matrix in ANP. European Journal of Operational Research, 213(1):246-259.

Fichtner, J. (1984). Some thoughts about the mathematics of the Analytic Hierarchy Process. Technical report, Institut für Angewandte Systemforschung und Operations Research, Universität der Bundeswehr München.

Genest, C., Lapointe, F., and Drury, S. W. (1993). On a proposal of Jensen for the analysis of ordinal pairwise preferences using Saaty's eigenvector scaling method. Journal of Mathematical Psychology, 37(4):575-610.

González-Díaz, J., Hendrickx, R., and Lohmann, E. (2014). Paired comparisons analysis: an axiomatic approach to ranking methods. Social Choice and Welfare, 42(1):139-169.

Henriet, D. (1985). The Copeland choice function: an axiomatic characterization. Social Choice and Welfare, 2(1):49-63.

Ho, W. (2008). Integrated analytic hierarchy process and its applications - A literature review. European Journal of Operational Research, 186(1):211-228.

Johnson, C. R., Beine, W. B., and Wang, T. J. (1979). Right-left asymmetry in an eigenvector ranking procedure. Journal of Mathematical Psychology, 19(1):61-64.

Kendall, G. and Lenten, L. J. A. (2017). When sports rules go awry. European Journal of Operational Research, 257(2):377-394.

Kóczy, L. Á. and Strobel, M. (2009). The invariant method can be manipulated. Scientometrics, 81(1):291-293.

Kułakowski, K. (2015). Notes on order preservation and consistency in AHP. European Journal of Operational Research, 245(1):333-337.

Landau, E. (1914). Über Preisverteilung bei Spielturnieren. Zeitschrift für Mathematik und Physik, 63:192-202. 
Lundy, M., Siraj, S., and Greco, S. (2017). The mathematical equivalence of the "spanning tree" and row geometric mean preference vectors and its implications for preference analysis. European Journal of Operational Research, 257(1):197-208.

Pérez, J. and Mokotoff, E. (2016). Eigenvector priority function causes strong rank reversal in group decision making. Fundamenta Informaticae, 144(3-4):255-261.

Perry, M. and Reny, P. J. (2016). How to count citations if you must. American Economic Review, 106(9):2722-2741.

Rabinowitz, G. (1976). Some comments on measuring world influence. Conflict Management and Peace Science, 2(1):49-55.

Rubinstein, A. (1980). Ranking the participants in a tournament. SIAM Journal on Applied Mathematics, 38(1):108-111.

Saaty, T. L. (1977). A scaling method for priorities in hierarchical structures. Journal of Mathematical Psychology, 15(3):234-281.

Saaty, T. L. (1980). The Analytic Hierarchy Process: Planning, Priority Setting, Resource Allocation. McGraw-Hill, New York.

Saaty, T. L. (2003). Decision-making with the AHP: Why is the principal eigenvector necessary. European Journal of Operational Research, 145(1):85-91.

Saaty, T. L. and Ozdemir, M. S. (2003). Why the magic number seven plus or minus two. Mathematical and Computer Modelling, 38(3-4):233-244.

Saaty, T. L. and Vargas, L. G. (2012). Models, Methods, Concepts $\&$ Applications of the Analytic Hierarchy Process. Springer Science \& Business Media, New York.

Tasnádi, A. (2008). The extent of the population paradox in the Hungarian electoral system. Public Choice, 134(3-4):293-305.

Vaidya, O. S. and Kumar, S. (2006). Analytic hierarchy process: An overview of applications. European Journal of Operational Research, 169(1):1-29.

van den Brink, R. and Gilles, R. P. (2009). The outflow ranking method for weighted directed graphs. European Journal of Operational Research, 193(2):484-491.

Vaziri, B., Dabadghao, S., Yih, Y., and Morin, T. L. (2018). Properties of sports ranking methods. Journal of the Operational Research Society, 69(5):776-787.

Williams, C. and Crawford, G. (1980). Analysis of subjective judgment matrices. Interim report R-2572-AF, Rand Corporation, Santa Monica.

Young, H. P. (1985). Monotonic solutions of cooperative games. International Journal of Game Theory, 14(2):65-72.

Zahedi, F. (1986). A simulation study of estimation methods in the analytic hierarchy process. Socio-Economic Planning Sciences, 20(6):347-354. 